NYU-TH-10/02/62

\title{
Field Theory for a Deuteron Quantum Liquid
}

\author{
Lasha Berezhiani, Gregory Gabadadze and David Pirtskhalava \\ Center for Cosmology and Particle Physics, Department of Physics, \\ New York University, New York, NY 10003, USA
}

\begin{abstract}
Based on general symmetry principles we study an effective Lagrangian for a neutral system of condensed spin-1 deuteron nuclei and electrons, at greater-than-atomic but less-than-nuclear densities. We expect such matter to be present in thin layers within certain low-mass brown dwarfs. It may also be produced in future shock-wave-compression experiments as an effective fuel for laser-induced nuclear fusion. We find a background solution of the effective theory describing a net spin zero condensate of deuterons with their spins aligned and anti-aligned in a certain spontaneously emerged preferred direction. The spectrum of low energy collective excitations contains two spin-waves with linear dispersions - like in antiferromagnets - as well as gapped longitudinal and transverse modes related to the Meissner effect - like in superconductors. We show that counting of the Nambu-Goldstone modes of spontaneously broken internal and space-time symmetries obeys, in a nontrivial way, the rules of the Goldstone theorem for Lorentz non-invariant systems. We discuss thermodynamic properties of the condensate, and its potential manifestation in the low-mass brown dwarfs.
\end{abstract}




\section{Introduction and Summary}

The field theory method is an extremely powerful tool for studying properties of matter and radiation at various scales. The subject of the present work is deuterium matter at greater-than-atomic, but much less-than-nuclear densities. At such densities the neutral deuterium atoms are disintegrated into charged spin-1 deuteron nuclei and electrons, with an average inter-particle separation being smaller than $1 \AA$ but much greater than $1 \mathrm{fm}$. At temperatures of interest in this work $\left(T \lesssim 10^{5} \mathrm{~K}\right)$, the electrons form a degenerate Fermi gas. Deuterons, on the other hand, can be regarded as point-like spin-1 charged particles, which as argued below, will condense beneath certain temperature forming a quantum liquid. A field theory description of this condensed state of deuterons is the main topic of the present work.

Recently, the condensation of charged spin-0 helium-4 nuclei at densities $\rho \sim$ $10^{6} \mathrm{~g} / \mathrm{cm}^{3}$ was studied using the effective field theory method [1]. Such a dense system is expected to be present in helium-core white dwarf stars. It was shown [2] that these stars will cool faster because of the condensation of helium- 4 nuclei (for a review see, $[3])$.

Here we develop an effective field theory approach to describe the deuteron quantum liquid. This is interesting for a few reasons: (i) Densities at which deuterons could condense are much lower than those for helium-4 nuclei; as argued below, at $\rho>100 \mathrm{~g} / \mathrm{cm}^{3}$, the deuteron condensate is expected to form upon gradual cooling of the system. (ii) The high-density deuteron matter is a promising fuel for laserinduced nuclear fusion. Hence, there has been a significant experimental interest in dense deuterium (as well as hydrogen) liquids (see [4] and references therein). Even though the present-day laboratory densities of shock-wave-compressed deuterium matter are two orders of magnitude below $100 \mathrm{~g} / \mathrm{cm}^{3}$, the experiments do see a transition to a liquid metallic state in which electrons get liberated from the atoms and the metallic conductivity is beginning to set in [4]. Given the importance of the subject one may hope the conditions necessary for reaching densities above $100 \mathrm{~g} / \mathrm{cm}^{3}$ could be achieved in not too far future. (iii) The deuteron matter with $\rho \sim 10^{3} \mathrm{~g} / \mathrm{cm}^{3}$ is expected to be present in localized regions within low temperature $\left(T \lesssim 10^{5} \mathrm{~K}\right)$ low-mass brown dwarfs. These are compact astrophysical objects that have not managed - because of their low mass and temperature - to ignite nuclear fusion (i.e., they are "failed stars"). As we will argue, these low-mass, cold brown dwarfs should contain the deuteron quantum liquid in a thin layer in their interiors. (iv) Theoretically, the deuteron quantum liquid seems to be a richer system than its helium-4 counterpart. Due to deuterons being spin-1, the spectrum of small fluctuations of the deuteron liquid exhibits properties similar to those of antiferromagnetic superconductors, as it will be shown in the paper. Moreover, the deuteron condensate represents an amusing example for which a consistency of counting of the Nambu-Goldstone modes in non-Lorentz-invariant theories [5] can be tested. (v) Our formalism can automatically be applied to condensates of other charged spin1 particles, or to neutral spin-1 particles with contact, and/or spin-spin, and/or 
magnetic dipole interactions, by taking various limits.

Before we turn to the field theory description in the next section, it is instructive to summarize the conditions for the condensation to take place.

At sufficiently high temperatures (but still significantly below the temperature of efficient thermo- and pycno-nuclear fusion), say at $T \lesssim 10^{5} \mathrm{~K}$, the system of deuterons would form a classical Bose gas, even though the electrons may already be in a quantum degenerate state. At lower temperatures, however, transitions of deuterons to different states is possible - they may crystallize, or alternatively, may form a quantum liquid.

Classical crystallization occurs at temperatures for which the Coulomb interaction energy of a pair of nuclei is about 180 times greater than the classical thermal energy of two degrees of freedom [6]. This leads to crystallization temperature for deuterons (see, [2] for recent discussions):

$$
T_{\text {cryst }} \simeq 1000 \cdot \rho^{1 / 3} K
$$

where density $\rho$ is in units of $g / \mathrm{cm}^{3}$. At high-enough densities, however, before crystallization temperature is reached from above, the deuterons may undergo transition into the charged condensate. This transition would take place around certain temperature $T_{c}$ for which the thermal de Broglie wavelength of the nuclei becomes greater than an average inter-particle separation between them. If $T_{c}$ significantly exceeds the crystallization temperature, then, before the system gets a chance to crystallize, the de Broglie wavelengths of the ions would begin to overlap leading to quantum-mechanical indistinguishability of the nuclei. If so, the standard arguments (see, e.g., [7]) suggest that the bosons would prefer to occupy one and the same quantum state, i.e., would prefer to condense. An estimate $[1,2]$ for the condensation temperature is

$$
T_{c} \simeq 350 \cdot \rho^{2 / 3} K
$$

The condensation temperature grows faster with $\rho$ than the crystallization temperature does ${ }^{1}$. In particular, comparing (1) and (2), we conclude that for densities greater than $100 \mathrm{~g} / \mathrm{cm}^{3}$, temperature $T_{c}$ significantly exceeds $T_{\text {cryst }}$, and hence deuteron condensation is favored upon gradual cooling of the system ${ }^{2}$.

In the next section, based on universal symmetry principles, we formulate an order-parameter effective field theory describing the deuteron condensate. We discuss the symmetry breaking patterns by the condensate, and count the broken generators. In section 3 we obtain the solution that describes a spin-0 state of condensed

\footnotetext{
${ }^{1}$ For a discussion of uncertainties in (1) and (2), which should not be affecting much our conclusions, see $[8,3]$.

${ }^{2}$ At yet lower temperatures, when the crystalline state also becomes available, there may exist spontaneous transition from the condensate to the crystalline state. However, this process is strongly suppressed [8].
} 
spin-1 deuterons, and discuss small fluctuations about that solution. Interestingly, the fluctuations contain a massive mode, which is well below the ultraviolet (UV) cutoff of the effective theory, as well as two massless modes with linear dispersions. The massive mode is a reflection of the Meissner effect, while the massless modes are similar to antiferromagnetic spin-waves. Hence, the condensate has properties similar to a antiferromagnetic superconductor, but has one order parameter. Moreover, the massless modes are Nambu-Goldstone (NG) bosons of spontaneously broken rotational symmetry. We discuss how counting of the NG modes agrees with the rules of Goldstone's theorem applied to non-Lorentz-invariant systems. In section 4 we study thermodynamic properties of a deuteron quantum liquid and briefly discuss its applications to low-mass and low-temperature brown dwarf interiors. Three appendices summarize various important technical details which, if placed in the main text, would overwhelm the presentation.

\section{Effective Field Theory and Symmetry Breaking}

The condensed state of deuterons discussed in the previous section will be described here by a complex vector order parameter $W_{i}(\mathbf{r}, t), i=1,2,3$, that can slowly vary at scales much greater than the average inter-deuteron separation, $d \sim J_{0}^{-1 / 3}$ (hereafter $J_{0}$ denotes the deuteron average number density, $J_{0} \sim 10^{-6} \mathrm{MeV}^{3}$ ). A non-zero background value of the order parameter -its expectation value in the state with a macroscopic occupation $\left\langle W_{i}(\mathbf{r}, t)\right\rangle$ - is a signature of the condensate. Small perturbations about the background, $\delta W_{i} \equiv W_{i}-\left\langle W_{i}\right\rangle$, describe long-wavelength collective excitations of the system.

In this section we will set up an effective Lagrangian for the order parameter $W_{i}(\mathbf{r}, t)$. Since the deuteron mass, $m_{H} \sim 1.876 \mathrm{GeV}$, is much greater than any energy/momentum scale that we'll be dealing with, the non-relativistic approximation in $m_{H}$ will be adopted (see Appendix A for the relativistic Lagrangian). The short-distance cutoff of the effective Lagrangian is set by the scale of interparticle separation $d \sim J_{0}^{-1 / 3}$. As we will see, the effective Lagrangian will capture massless, as well as massive collective excitations of the system. The latter have masses $\sim g\left(J_{0} / m_{H}\right)^{1 / 2}$, where $g$ denotes the gauge coupling constant. Because of the hierarchy $J_{0}^{1 / 3} \ll m_{H}$, the above mass scale is parametrically supressed in comparison with the UV cutoff of the theory, $d^{-1} \sim J_{0}^{1 / 3}$, and hence, can be calculated reliably within the effective Lagrangian approach.

The effective Lagrangian has to be invariant w.r.t. all the internal and space-time symmetries appropriate for the physical system at hand. In particular, it should possess the local $U(1)_{E M}$ symmetry of electromagnetism, the global subgroup of which guarantees the electric charge conservation. It should also posses a global $U(1)_{s}$ symmetry w.r.t. $W_{i}(\mathbf{r}, t) \rightarrow W_{i}(\mathbf{r}, t) e^{i \beta}$ with an arbitrary constant $\beta$, as the latter guarantees conservation of the number of deuterons. The above two global symmetries, once put together, imply that the electron number is also conserved. 
As to space-time symmetries, the Lagrangian should be invariant w.r.t. spatial rotations and reflections represented by the $O(3)$ group; it should also be Galilean invariant. Finally, in the limit when all the relevant coupling constants are set to zero, the equations of motion should reduce to the ordinary Schrödinger equation for the order parameter $W_{i}$.

The Lagrangian that satisfies all the above conditions and describes the bosonic sector of the theory reads as follows [we use the "mostly-minus" metric convention]:

$$
\begin{aligned}
\mathcal{L}_{1}= & -\frac{1}{4} F_{\mu \nu} F^{\mu \nu}-\frac{i}{2}\left(W^{* i} D_{0} W_{i}-W^{i}\left(D_{0} W_{i}\right)^{*}\right)-\frac{1}{2 m_{H}}\left|D^{i} W_{j}\right|^{2}- \\
& -\frac{\lambda_{q}}{8 m_{H}^{2}}\left(W^{* i} W_{i}\right)^{2}-\frac{\lambda_{s}}{8 m_{H}^{2}}\left(\epsilon^{i j k} W_{j}^{*} W_{k}\right)^{2}-i g \frac{(1-\kappa)}{2 m_{H}} W_{i}^{*} W_{j} F^{i j} .
\end{aligned}
$$

The covariant derivative is defined as $D_{\mu}=\partial_{\mu}-i g A_{\mu}, g$ is a charge of $W$. The effective mass of a deuteron in the interacting system is denoted by $m_{H}$. We will assume that this mass - being the highest scale - does not get renormalized too much by interactions and set $m_{H} \sim 2 \mathrm{GeV}$. The parameters $\kappa, \lambda_{s}, \lambda_{q}$ are dimensionless coupling constants. The last term in the Lagrangian parametrizes the effects of the magnetic moment of charged deuteron nuclei for which we included the tree-level as well as loop-generated terms (the latter is parametrized by $\kappa$ ). Furthermore, the $\lambda_{s^{-}}$term describes the spin-spin interaction, while the $\lambda_{q^{-}}$term parametrizes the contact repulsion of the deuterons ${ }^{3}$.

We have included in (3) only leading order terms. There are higher dimensional terms in the effective Lagrangian that are consistent with the symmetries imposed. A systematic way to include those terms is to turn to the canonically normalized fields (replace $W \rightarrow W \sqrt{2 m_{H}}$ ), and then write down the higher dimensional operators suppressed by appropriate powers of the short distance (UV) cutoff, $d^{-1} \sim J_{0}^{1 / 3}$. Because of the hierarchy $J_{0}^{1 / 3} \ll m_{H}$, the higher dimensional terms will not play a significant role for the low energy excitations of the system and will be ignored.

In addition to (3) the full theory also includes terms describing electrons of some effective mass $m_{e}$, for which we'll be using the Dirac Lagrangian

$$
\mathcal{L}_{2}=i \bar{\psi} \gamma^{\mu}\left(\partial_{\mu}+i g A_{\mu}\right) \psi-m_{e} \bar{\psi} \psi
$$

The total Lagrangian reads as follows: $\mathcal{L}^{N R}=\mathcal{L}_{1}+\mathcal{L}_{2}$. For the reasons discussed above, we will also ignore higher dimensional terms which couple electrons directly to the order parameter (an example being $W^{*} W \bar{\psi} \psi$ ). Even though we retain the Dirac Lagrangian in the canonical form (4), the electrons themselves are heavy and decoupled from the low-energy dynamics. What is not decoupled are the gapless excitations of the electrons near the Fermi surface. To begin with, we treat the

\footnotetext{
${ }^{3}$ Note that in a "fundamental theory" the $\kappa, \lambda_{s}, \lambda_{q}$-terms need not be included in the tree-level Lagrangian as they'll be generated via loop corrections. In the effective Lagrangian, since we're not going to be calculating the loops, these terms were introduced with arbitrary coefficients in (3).
} 
fermionic fluctuations in the Thomas-Fermi (TF) approximation, although this will be refined later on to include a loop correction due to those near-the-Fermi-surface gapless modes. In the TF approximation we average the fermionic four-current as follows: $J^{\mu} \equiv\left\langle\bar{\psi} \gamma^{\mu} \psi\right\rangle=J_{0} \delta_{0}^{\mu}$. This sets up a nonzero electron number-density $J_{0}$ in the ground state. Note that this state does not break the global $U(1)$ symmetry responsible for the fermion number conservation.

As we will show in the next section the solution

$$
\left\langle A_{\mu}\right\rangle=\frac{\lambda_{q} v^{2}}{2 m_{H}} \delta_{\mu}^{0}, \quad\left\langle W_{\mu}\right\rangle=(0,0,0, v)
$$

where $v \equiv \sqrt{J_{0} / 2 m_{H}}$, represents a consistent classical background of the theory. It describes a state of condensed nuclei with zero net spin, in which the individual deuteron spins are aligned or anti-aligned with the preferred direction set by a vector $\mathbf{v}=(0,0, v)$. Such a spin alignment, and the resulting spin-waves discussed in the next section, are similar to those in antiferromagnetic materials. On the other hand, the properties of the electromagnetic field in this substance will be shown to be similar to those in superconductors.

Note that a nonzero expectation value of $A_{0}$ in (5) shifts the value of Fermi energy, however, this correction is typically of the order $\sim \lambda_{q} 10^{-12} \mathrm{MeV}$, and will be ignored.

Before turning to the next section, let us discuss the pattern of symmetry breaking by the background (5) in a few specific cases:

Case " $a$ ": $\lambda_{q} \neq 0, \lambda_{s} \neq 0, \kappa \neq 1$. The internal part of the symmetry group $G$ of the theory is given by $G_{i n t}=U(1)$, whereas the relevant-to-us part of the spacetime symmetry consists of the group of reflections and spatial rotations $G_{s t}=O(3)_{s}$ (the Galilean and space-time translation subgroups of $G$ are left unbroken by the condensate, and are ignored in the further discussions). Upon vector condensation (5), the symmetry group is broken down to spatial $O(2)_{s}$, corresponding to rotations and reflections in the plane perpendicular to $\mathbf{v}=(0,0, v)$. Therefore, $U(1) \times O(3)_{s} \rightarrow$ $O(2)_{s}$, and the number of spontaneously broken continuous symmetry generators ends up being equal to 3 .

Case " $b ": \lambda_{q} \neq 0, \lambda_{s} \neq 0, \kappa=1$. The internal symmetry group gets enhanced by an additional factor of $O(3)_{i}$ corresponding to a simultaneous internal rotation of $W_{i}$ and $W_{i}^{*}$ by orthogonal three-by-three matrices. The diagonal part of $O(3)_{i} \times O(3)_{s}$ gets broken by the vacuum (5) to the diagonal $O(2)_{d}$, whereas the orthogonal combination $O(3)_{a}$ remains unbroken. This leads to the following pattern of symmetry breaking $U(1) \times O(3)_{i} \times O(3)_{s} \rightarrow O(3)_{a} \times O(2)_{d}$, and consequently to three broken generators.

Case "c": $\lambda_{q} \neq 0, \lambda_{s}=0, \kappa=1$. Internal symmetry is enhanced further to $G_{\text {int }}=O(6)$. The symmetry breaking pattern is then $O(6) \times O(3)_{s} \rightarrow O(5) \times O(3)_{l i n}$, where $O(3)_{\text {lin }}$ is a linear combination of a subgroup of $O(6)$ and $O(3)_{s}$. This results in five broken generators. 
The Lagrangian (3) describes a real vector field $A_{\mu}$ with two dynamical components, and a complex massive vector field $W_{i}$ with six components three of which are heavy degrees of freedom (mass $\propto m_{H}$ ) which should be ignored; this leaves altogether five potentially light degrees of freedom. Irrespective of the Case "a", "b" or "c" described above, the $U(1)$ electromagnetic symmetry is spontaneously broken by the condensate, hence, the longitudinal NG boson will become massive to serve as a helicity-0 component of a massive photon. Therefore, two NG bosons should remain and satisfy the counting rules for the different symmetry breaking patterns given in Cases "a", "b" or "c". At a first sight this seems unlikely, however, as we will see in the next section, that the dynamically obtained NG modes will obey the rules of counting of the Goldstone theorem for Lortentz-non-invariant models [5]; these rules are quite different from those in relativistic theories.

Last but not least, in this and the next section we're discussing the effective Lagrangian at zero temperature (finite temperature effects in charged scalar condensate have been recently studied in [9]), even though what we actually have in mind is temperature much lower than the deuteron condensation temperature, but higher than the room temperature. A caution has to be exercised in this regard - at a certain temperature $T_{e} \ll 10^{-6} K$ (see the next section) there will be additional phase transition in which the Cooper pairs of near-the-Fermi-surface electrons will form via the Kohn-Luttinger mechanism [10] (see, [3] for a brief discussion of this effect in the presence of the BE condensate). Then, below $T_{e}$ a new NG boson will emerge as a result of spontaneously broken fermion-number symmetry triggered by the electron-electron order parameter. In conventional superconductivity an analogous NG Boson combines with a photon to form a massive photon. In the present case, however, this role is already played by the longitudinal mode associated with spontaneously broken $U(1)_{s}$ boson-number symmetry discussed above ${ }^{4}$. On the other hand, since $T_{e}$ is close to zero for any system discussed in this work we'd expect temperature to be way above $T_{e}$. Therefore, in our discussions we will not invoke the Cooper pairing mechanism with the fermion-number breaking condensate, and will ignore the finite temperature effects.

\section{Background and its Small Fluctuations}

Below we derive the background solution and study fluctuations about it. It is convenient to use a slightly different form of the Lagrangian (3) in which we "integrate in" an auxiliary field $W_{0}$, and also perform the following rescalings of the fields, $W \rightarrow W \sqrt{2 m_{H}}, A \rightarrow A / g$. Then, the part of the Lagrangian relevant for

\footnotetext{
${ }^{4}$ In reality one should consider linear combinations of these two NG bosons; one of the combinations will become massive and another one will remain massless. However, since $g J_{0} / m_{H} \gg T_{e}^{2}$, the mixing effects are negligible.
} 
studying the bosonic background and its fluctuations reads as follows:

$$
\begin{gathered}
\mathcal{L}_{1}=-\frac{1}{4 g^{2}} F_{\mu \nu} F^{\mu \nu}-\frac{1}{2} \Sigma_{i j}^{*} \Sigma^{i j}+i m_{H} W^{* i} \Sigma_{i 0}-i m_{H} W^{i} \Sigma_{i 0}^{*}+m_{H}^{2} W_{0}^{*} W^{0}- \\
-J_{\mu} A^{\mu}-\frac{\lambda_{q}}{2}\left(W^{* i} W_{i}\right)^{2}-\frac{\lambda_{s}}{2}\left(\epsilon^{i j k} W_{j}^{*} W_{k}\right)^{2}+i \kappa W^{* i} W^{j} F_{i j}
\end{gathered}
$$

where $\Sigma_{i j}=D_{i} W_{j}-D_{j} W_{i}$ denotes the gauged field strength of $W$, and the fermions have been treated in the mean-field approximation, as mentioned before ${ }^{5}$. There are no time derivatives of the $W_{0}$ field in (6), and as noted before, it is an auxiliary variable which can be integrated out to get back the Lagrangian (3).

Equations of motion for the fields $W_{0}^{*}, W_{i}^{*}, A_{0}, A_{i}$ are straightforward to obtain from (6). They read respectively as follows:

$$
\begin{array}{r}
i m_{H} D^{i} W_{i}+m_{H}^{2} W_{0}=0 \\
D^{j} \Sigma_{j i}-i m_{H} D_{0} W_{i}+i m_{H} \Sigma_{i 0}-\left(\lambda_{q}+\lambda_{s}\right)\left(W^{* j} W_{j}\right) W_{i}+\lambda_{s}\left(W^{j} W_{j}\right) W_{i}^{*}+ \\
i \kappa W^{j} F_{i j}=0, \\
\frac{1}{g^{2}} \partial^{j} F_{j 0}-2 m_{H} W^{* i} W_{i}=J_{0}, \\
\frac{1}{g^{2}} \partial^{\mu} F_{\mu i}+i W^{* j} \Sigma_{j i}-i W^{j} \Sigma_{j i}^{*}+m_{H}\left(W_{i}^{*} W_{0}+W_{i} W_{0}^{*}\right)-i \kappa \partial^{j}\left(W_{j}^{*} W_{i}-W_{i}^{*} W_{j}\right)=0 .
\end{array}
$$

It is not difficult to check that these equations have the following classical solution

$$
A_{\mu}-\partial_{\mu} \alpha=\frac{\lambda_{q} v^{2}}{2 m_{H}} \delta_{\mu}^{0}, \quad W_{\mu}=\left(0, e^{i \alpha} \mathbf{v}\right)
$$

where $\mathbf{v}$ is an arbitrarily-directed real vector with the norm $v \equiv \sqrt{J_{0} / 2 m_{H}}$, and $\alpha$ is an overall phase of the vector field $W_{\mu}$. As discussed in the previous section, we choose our background solution to be:

$$
\left\langle A_{\mu}\right\rangle=\frac{\lambda v^{2}}{2 m_{H}} \delta_{\mu}^{0}, \quad\left\langle W_{\mu}\right\rangle=(0,0,0, v)
$$

This solution describes a state of a large occupation number of bosons, the net electric charge of which is compensated by the electron charge density $J_{0}$. The quantities that acquired the vacuum expectation values are gauge-invariant, as can be seen from (7). The gauge-choice given by (8), on the other hand, corresponds to the unitary gauge.

To study the spectrum of small perturbations, it is useful to work in terms of the gauge-invariant variables

$$
A_{\mu}-\partial_{\mu} \alpha_{3}, \quad\left|W_{3}\right|, \quad W_{0,1,2} e^{-i \alpha_{3}}
$$

\footnotetext{
${ }^{5} \mathrm{~A}$ derivation of (6) as a non-relativistic limit of a relativistic theory in given in Appendix B.
} 
where $\alpha_{3}$ denotes the phase of $W_{3}$ (the same results would have been obtained by choosing the unitary gauge). As mentioned before, we treat the fermionic fluctuations in the Thomas-Fermi approximation: This can be accounted for in the equations of motion by increasing the coefficient of the $A_{0}$ perturbation by $m_{0}^{2} / g^{2} \equiv\left(3 \pi^{2} J_{0}\right)^{2 / 3} / \pi^{2}$ in the ultra-relativistic regime for the electron Fermi-gas, or by $m_{0}^{2} / g^{2} \equiv m_{e}\left(3 \pi^{2} J_{0}\right)^{1 / 3} / \pi^{2}$ in the non-relativistic regime.

The equations of motion for the excitations on the condensate, as well as the procedure of obtaining the linear spectrum are briefly summarized in Appendix C. The calculations are straightforward but very tedious. We find that the dispersion relations for the relevant, low-energy modes are given by the following:

Longitudinal polarization of the massive photon. After symmetry breaking, one combination of the fields becomes the longitudinal mode of the massive photon, with the dispersion relation given by

$$
\omega_{1}^{2} \simeq 2 v^{2} g^{2}\left(1+\frac{\mathbf{k}^{4}+2 \lambda_{q} v^{2} \mathbf{k}^{2}}{8 g^{2} m_{H}^{2} v^{2}}\right) .
$$

An identification of the corresponding mode is done by invoking the following arguments: first, we see that this dispersion is independent of the spin-spin interaction constant $\lambda_{s}$, as well as the magnetic moment interaction constant $(1-\kappa)$, suggesting that this is a longitudinal mode. Second, in the limit of zero electromagnetic coupling, $g \rightarrow 0$, the dispersion relation for small $\mathbf{k}$ is linear in $\mathbf{k}$ and is determined by the "contact repulsion" constant $\lambda_{q}$, as it should be for a longitudinal mode ${ }^{6}$. Third, if in addition we take the limit $\lambda_{q} \rightarrow 0$ we obtain the dispersion relation for a small fluctuation above a Bose-Einstein (BE) condensate of free particles, $\omega_{1} \simeq \mathbf{k}^{2} / 2 m_{H}$.

Transverse polarizations of the photon. A complete set of dispersion relations for the two transverse modes of the photon are given in Appendix C by $\omega_{2,3}$ in (C-VII). In the limit when all the coupling constants are switched off we obtain ordinary photon dispersions. In the gauge theory the transverse modes acquire mass gaps, which can easily be seen by setting $\mathbf{k} \rightarrow 0$. Expanding $\omega_{2,3}$ in (C-VII) in powers of the momentum for which $|\mathbf{k}| \ll g v$, one obtains

$$
\begin{gathered}
\omega_{2}^{2} \simeq 2 g^{2} v^{2}+\mathbf{k}^{2}+\frac{(1-\kappa)^{2}}{4 m_{H}^{2}} \mathbf{k}^{4}, \\
\omega_{3}^{2} \simeq 2 g^{2} v^{2}+\mathbf{k}^{2}+\frac{(1-\kappa)^{2}}{4 m_{H}^{2}} \frac{(\mathbf{k} \cdot \mathbf{v})^{2}}{v^{2}} \mathbf{k}^{2} .
\end{gathered}
$$

Hence, the transverse modes are massive with the value of the mass coinciding with that of the longitudinal mode (10). A seeming independence of the last terms in the dispersion relations (11), (12) from the gauge coupling constant is due to the specific nature of the expansion $|\mathbf{k}| \ll g v$ - the dependence on $1 / g v$ shows up in

\footnotetext{
${ }^{6}$ In the denominator of the second term in (10) we ignored terms proportional to $m_{0}^{2} \mathbf{k}^{2}$ and $\lambda_{q} v^{2} m_{0}^{2}$. These terms are always sub-dominant to the term retained in the denominator.
} 
higher order terms. The exact expressions (C-VII) do no exhibit any pathological behavior.

In the opposite limit $|\mathbf{k}| \gg g v$, one may perform the $1 / m_{H}$ expansion, leading to the following dispersions

$$
\begin{gathered}
\omega_{2}^{2} \simeq 2 g^{2} v^{2}+\mathbf{k}^{2}+2 g^{2} v^{2} \frac{(1-\kappa)^{2}}{4 m_{H}^{2}} \mathbf{k}^{2}, \\
\omega_{3}^{2} \simeq 2 g^{2} v^{2}+\mathbf{k}^{2}+2 g^{2} v^{2} \frac{(1-\kappa)^{2}}{4 m_{H}^{2}} \frac{(\mathbf{k} \cdot \mathbf{v})^{2}}{v^{2}} .
\end{gathered}
$$

As we see, propagation of the transverse modes in general is affected by the magnetic moment interaction. However, $\omega_{3}$ depends on the preferred direction of the spinantispin alignment set by the vector $\mathbf{v}$, while $\omega_{2}$ does not. As a result, for momenta perpendicular to the preferred direction, frequency $\omega_{3}$ is independent of $(1-\kappa)$. On the other hand, when the direction of propagation coincides with the preferred direction, the expressions for $\omega_{2}$ and $\omega_{3}$ coincide too; this is a manifestation of the remaining rotational symmetry in the plane perpendicular to this direction.

$N G$ bosons of broken rotational symmetries. The two massless NG modes constitute the rest of the spectrum, their dispersion relations being given by $\omega_{4,5}$ in (C-VII). In the zero coupling limit these reduce to the dispersion relations of small fluctuations over the BE condensate of free bosons, $\omega_{4,5} \simeq k^{2} / 2 m_{H}$. In the interacting theory we perform the expansions similar to the ones used for the transverse modes, and obtain

$$
\begin{gathered}
\omega_{4}^{2} \simeq \frac{\mathbf{k}^{4}}{4 m_{H}^{2}}+\lambda_{s} \frac{\mathbf{k}^{2} v^{2}}{2 m_{H}^{2}}-\frac{(1-\kappa)^{2}}{4 m_{H}^{2}} \mathbf{k}^{4}, \\
\omega_{5}^{2} \simeq \frac{\mathbf{k}^{4}}{4 m_{H}^{2}}+\lambda_{s} \frac{\mathbf{k}^{2} v^{2}}{2 m_{H}^{2}}-\frac{(1-\kappa)^{2}}{4 m_{H}^{2}} \frac{(\mathbf{k} \cdot \mathbf{v})^{2}}{v^{2}} \mathbf{k}^{2},
\end{gathered}
$$

in the $|\mathbf{k}| \ll g v$ limit, while for the $|\mathbf{k}| \gg g v$ case we obtain

$$
\begin{gathered}
\omega_{4}^{2} \simeq \frac{\mathbf{k}^{4}}{4 m_{H}^{2}}+\lambda_{s} \frac{\mathbf{k}^{2} v^{2}}{2 m_{H}^{2}}-2 g^{2} v^{2} \frac{(1-\kappa)^{2}}{4 m_{H}^{2}} \mathbf{k}^{2}, \\
\omega_{5}^{2} \simeq \frac{\mathbf{k}^{4}}{4 m_{H}^{2}}+\lambda_{s} \frac{\mathbf{k}^{2} v^{2}}{2 m_{H}^{2}}-2 g^{2} v^{2} \frac{(1-\kappa)^{2}}{4 m_{H}^{2}} \frac{(\mathbf{k} \cdot \mathbf{v})^{2}}{v^{2}} .
\end{gathered}
$$

A few comments are in order here: (i) As in the case of the transverse modes, seeming independence of the leading terms in NG dispersion relations (15), (16) from the gauge coupling constant is an artifact of the expansion in $|\mathbf{k}| / g v \ll 1$. The spectrum is stable for $\kappa \in[0,2]$ and $\lambda_{s} \geq 0$. (ii) The $\lambda_{s}$-dependent quadratic terms in the NG dispersion relations are due to the spin-spin interaction term $\left(\lambda_{s}\right.$-term) in the original Lagrangian (6). (iii) The last, $(1-\kappa)$-dependent terms in (17) and (18), are due to magnetic moment interactions of oppositely aligned spins of charged deuterons. Since these interactions are attractive, the minus sign in front of the last 
terms in (17) and (18) appears. In the exact expressions (C-VII) these negative terms can never overcome the positive ones, and the spectrum is stable. (iv) The dependence on the gauge coupling constant seems to disappear from the dispersions of the NG modes for $\kappa=1$. However, in the system of deuterons and electrons the effective constant of the spin-spin interactions, $\lambda_{s}$, and the anomalous magnetic moment of deuteron, $\kappa$, will be proportional to positive powers of the gauge coupling. (v) As in the case of the transverse modes, for momenta aligned with the preferred direction, the dispersion relations for the two NG bosons coincide, as a reflection of the remaining $O(2)$ symmetry group.

All the described properties above suggest that the obtained gapless states are NG bosons of spontaneously broken rotational symmetry. Their properties are very similar to spin-waves in antiferromagnets; in the present case they emerge due to spins of the charged nuclei.

It is time now to check a consistency of counting of the NG bosons with Goldstone's theorem for Lorentz non-invariant systems. This counting is rather different from that in relativistically invariant theories, and goes as follows [5]: if one denotes the number of broken generators of continuous global symmetry by $q$, then the number of NG bosons should satisfy the following inequality

$$
q \leq n_{1}+2 n_{2}
$$

where $n_{1}$ is the numbers of NG modes with frequencies proportional to an odd power of momentum in the long wavelength limit, (e.g., $\omega \propto|\mathbf{k}|$; these are called "type I"), while $n_{2}$ is the numbers of NG modes with frequencies proportional to an even power of momentum in that limit (e.g., $\omega \propto|\mathbf{k}|^{2}$; called "type II").

One can easily check, that (19) is remarkably satisfied by the NG modes found for the cases "a", "b", and "c" discussed at the end of the previous section. In the Case "a" or "b", there are three broken generators one of which is Higgsed, hence, $q=2$. This is matched by two type I NG modes, which saturate the inequality (19). If we impose a further condition that $\lambda_{s}=0$ - as in the Case "c" - then we end up with 5 broken generators one of which is Higgsed, leading to $q=4$. On the other hand, both NG bosons found above end up being of type II in this limit, hence the inequality (19) is saturated again.

One more interesting example is that of Case "a" with an additional condition $\lambda_{s}=0$. The symmetry breaking pattern in this case is identical to that of Case "a". However, the dispersion relations for the NG modes, (15) and (16), become of type II, i.e, we get $n_{1}=0$ and $n_{2}=2$. Clearly, in this case the inequality (19) is not saturated, but is satisfied.

As discussed at the end of the previous section, there will be one more longitudinal NG mode that emerges at extremely low temperatures (very close to the absolute zero) as a result of spontaneous breaking of the femion-number symmetry due to Cooper pairing of the near-the-Fermi-surface electrons via the Kohn-Luttinger mechanism. 
We end this section by considering more refined effects of the near-the-Fermisurface electrons. So far we have taken these effects into account in the ThomasFermi approximation. This approximation, however, fails to capture one of the most important properties of the fermion system, namely that the gapless modes near the Fermi surface can give rise to long range interactions for charged particles via the loop effects.

One of the manifestations of this is the well-known Friedel potential [11], and the Kohn-Luttinger effect itself. It is important to see how these considerationstraditionally done for a system with fermions only - are affected by the presence of the spin-1 charge condensate (for spin-0 see [1]).

To study these effects we consider a potential between two static charges (e.g., two impurities, such as helium-3 or helium-4 nuclei). The zero-zero component of the frequency-independent momentum-space Green's function in our case reads as follows (for simplicity, we take $\lambda_{q}=0$ ):

$$
G(\omega=0, \mathbf{k}, \lambda=0)=\frac{\mathbf{k}^{2}}{\mathbf{k}^{4}+4 M^{4}},
$$

where $M \equiv\left(2 g^{2} m_{H}^{2} v^{2}\right)^{1 / 4}$. In the coordinate space this would give rise to an exponentially suppressed oscillating potential $\left(e^{-M r} / r\right) \cos (M r)$. We now include the contribution of the electron via one loop vacuum polarization diagram. This leads to two effects [11]: (i) the pole of the Green's function gets shifted (this is what leads to the Debye screening, and is the effect captured in the Thomas-Fermi approximation by the parameter $m_{0}^{2}$ ); (ii) the gapless excitations near the Fermi surface, as in the case of the Friedel potential, lead to branch-cuts in the complex $|\mathbf{k}|$-plane. These branch-cuts give rise to the long range piece in the position-space potential, which in the case with relativistic fermions (taken for simplicity), ends up being similar to the one obtained in [1]

$$
V_{\text {stat }}=\alpha_{\mathrm{em}} Q_{1} Q_{2}\left(\frac{e^{-M r}}{r} \cos (M r)+\frac{4 \alpha_{\mathrm{em}}}{\pi} \frac{k_{F}^{5} \sin \left(2 k_{F} r\right)}{M^{8} r^{4}}\right) \text {. }
$$

Here $Q_{1,2}$ denote the charges of static sources (impurities), $\alpha_{e m}$ is the fine structure constant and $k_{F^{-}}$Fermi momentum of the system.

As in the case of the charged spin- 0 condensate, the obtained long-range potential is suppressed in comparison with the standard Friedel potential. The reason for this suppression is the subtraction due to an exchange of the longitudinal polarization of the photon, as discussed in detail in [1].

The potential (21) is sign-indefinite and one may wonder whether its attractive part could trigger some instability near the Fermi surface to form Cooper pairs. In fact, the analogous oscillatory behavior in the interaction potential between electrons is what gives rise to the Kohn-Luttinger effect. However, as we see the potential is too small - a "typical" value for it is $10^{-16} \mathrm{MeV} \sim 10^{-6} \mathrm{~K}$, and therefore any tiny finite temperature will wash-out the effects of this potential. This small value 
of the potential is also the reason for the temperature of the Kohn-Luttinger type transition to be very low - it should be that $T_{e} \ll 10^{-6} K$.

\section{Some Properties of the Condensate}

Already at densities $\rho \sim 10^{3} \mathrm{~g} / \mathrm{cm}^{3}$ (which is well above atomic densities for deuterium), the temperature of condensation significantly exceeds that of crystallization (the critical density, at which $T_{c} \simeq T_{\text {cryst }}$ is about an order of magnitude lower). Hence, according to the arguments given in section 1 , at these densities the charged deuteron condensate is expected to form at $T<T_{c} \sim 3.5 \cdot 10^{4} \mathrm{~K}$.

In order to study thermodynamics of this substance at $T \ll T_{c}$, we need an expression for the temperature dependence of its specific heat (per ion). The latter will be determined by the massless NG modes ${ }^{7}$. The expression for the specific heat (in the units of the Boltzmann constant) is readily obtained from

$$
c_{v}=\frac{1}{(2 \pi)^{3} J_{0}} \int^{k_{c}} d^{3} k \frac{\omega^{2} e^{\omega / T}}{\left(e^{\omega / T}-1\right)^{2} T^{2}} .
$$

Here $k_{c} \equiv 2 \pi / d$ represents the natural momentum cutoff in the effective theory. For densities mentioned above, $k_{c} \sim 10^{-1} \mathrm{MeV}$. At the momenta close to the cutoff, the dominant contribution in the NG dispersion relation is given by the first terms in (17)-(18), which are of the order $\omega_{4,5} \sim 10^{-6} \mathrm{MeV} \simeq 10^{4} \mathrm{~K}$.

We note that for the momenta greater than $k_{0}=\sqrt{\lambda_{s}} \cdot 10^{-5} \mathrm{MeV}$, the $k^{2} / 2 m_{H}$ term still dominates in the NG dispersion relations for most of the integration range in (22). A simple numerical analysis shows, that the contribution from the momentum domain $k \in\left(0, k_{0}\right)$ is negligible as long as $T \gg \lambda_{s} v^{2} / m_{H} \sim \lambda_{s} \cdot 10^{-3} \mathrm{~K}$, which is assumed to be the case here.

Therefore, the thermodynamics of the NG gas is determined by the free particle dispersion in the temperature range at hand, $\lambda_{s} \cdot 10^{-3} \mathrm{~K}<T<10^{4} \mathrm{~K}$. Using these estimates, we derive the expression for the specific heat per ion

$$
c_{v}=\frac{1}{J_{0}}\left(\frac{2 m_{H} T}{\pi}\right)^{\frac{3}{2}} \frac{15 \zeta(5 / 2)}{32} .
$$

At temperatures $T \ll \lambda_{s} v^{2} / m_{H} \simeq \lambda_{s} \cdot 10^{-3} \mathrm{~K}$, on the other hand, $c_{v}$ is determined by the $\lambda_{s}$ - term in the NG dispersion relations. Specific heat per deuteron in the ultra-low temperature limit therefore, is given by

$$
c_{v}=\frac{16 \pi^{2}}{15 \lambda_{s}^{3 / 2}} \frac{m^{9 / 2} T^{3}}{J_{0}^{5 / 2}} .
$$

\footnotetext{
7The gapped modes - two transverse and one longitudinal- are effectively "decoupled" due to their mass $\sqrt{2} g v \sim 5 \cdot 10^{4} \mathrm{~K}$ being greater than the condensation temperature, while fermion contribution to the specific heat is negligible.
} 
This is true as long as temperature is above the point $T_{e}$ where the Cooper pairing due to the Kohn-Luttinger (KL) effect will take place. Below this point there will be an additional NG boson contributing to the expression for specific heat. The latter will still scale as $T^{3}$. Above or below the temperature of the KL transition, however, we expect that the heat transfer in deuteron condensate will be dominated by temperature-waves ("second sound") in the two-component phonon-superfluid medium.

The densities of interest in the present work are expected to be present in certain astrophysical systems, namely in sub-stellar objects with masses low enough not to be able to fuse deuterium. An example is a low-temperature $\left(T \lesssim 10^{5} \mathrm{~K}\right)$, low-mass $\left(M<13 \cdot M_{\text {Jupiter }}\right)$ brown dwarf. Such an object is supported by the electron Fermi degeneracy pressure working against gravity. An average interior density in the core could be close to $\sim 10^{3} \mathrm{~g} / \mathrm{cm}^{3}$ and the radius - of the order of Jupiter's radius ${ }^{8}$. The abundances of various nuclei (protons, deuterons and helium) in the core of such a dwarf are expected to trace the cosmological abundances of the corresponding chemical elements. In particular, the fractions of protons, deuterons and helium nuclei in the core should be similar to the fractions of the corresponding chemical elements in Jupiter's core $N_{\text {protons }} \simeq 30 \cdot N_{\text {helium }} \simeq 10^{5} \cdot N_{\text {deuteron. }}$. Furthermore, we assume a non-convective interior, and the chemical stratification of the elements (separation of chemical elements) due to rotation of the object. Under these conditions the very center of the core would dominantly consist of helium sub-core of radius $\sim R_{\text {Jupiter }} / 2$, surrounded by a thin deuteron layer of $\sim 2 \mathrm{~km}$ width, which is topped by a thick layer of protons, $R_{\text {proton }} \sim R_{\text {Jupiter }} / 2$. For temperatures around $10^{4} \mathrm{~K}$ and densities at hand, the mean free path of the deuteron nuclei could be estimated to be $\sim 30 \mathrm{~m}$, suggesting that the smearing of the thin deuteron layer into the upper proton and lower helium layers is negligible. Also, the thermo- and pycno-nuclear fusion rates are small. Hence, we got conditions appropriate for condensation of the deuteron nuclei. The state of the core of this brown dwarf can be described as follows: in the sub-core the helium nuclei are crystallized; this is followed by a thin layer of a deuteron quantum liquid, which is topped by a gas of protons that is about to become quantum at slightly lower temperatures. Such an arrangement in the core should impact rotational properties of the brown dwarf. In particular, the rotation of the proton layer should not be expected in general to be in a phase with the rotation of the solid helium core, and therefore, certain irregularities in rotation might emerge.

\section{Acknowledgments}

We'd like to thank Yosi Gelfand, Andy Kent and Arkady Vainshtein for useful discussions. The work of GG is supported by NSF grant PHY-0758032. LB and DP

\footnotetext{
${ }^{8}$ For simplicity, we consider a homogeneous interior. This is a good approximation up to a factors of a few, as one can show, based on a nonrelativistic polytrope model [12].
} 
are supported respectively by MacCracken and James Arthur Graduate Fellowships at NYU.

\section{Appendix A. Relativistic Description of the Condensate}

For a relativistic description of the system of deuterons in the background of electrons, we use an order parameter $V_{\mu}$ (an early treatment is given in [13]; for modern discussion, see [14]). The action should possess a local $U(1)_{E M}$, as well as Poincare invariances. The dynamics of the system is therefore described by the following effective Lagrangian

$$
\mathcal{L}=-\frac{1}{4 g^{2}} F_{\mu \nu} F^{\mu \nu}-\frac{1}{2} G_{\mu \nu}^{*} G^{\mu \nu}+m_{H}^{2} V_{\mu}^{*} V^{\mu}-J_{\mu} A^{\mu}
$$

Here $G_{\mu \nu}=D_{\mu} V_{\nu}-D_{\nu} V_{\mu}$ is the field strength (covariant derivative being defined as $D_{\mu}=\partial_{\mu}-i A_{\mu}$ ), and $J_{\mu}$ represents the averaged fermionic current. It should be noted, that in this notation $A_{\mu}$ is rescaled by a factor of $g$, as compared to the physical normalization of the photon field.

There exist three additional dimension-four, gauge-invariant operators which could contribute to the effective Lagrangian. These are the quartic self-couplings and magnetic dipole interactions of the form

$$
\mathcal{L}^{\prime}=-\frac{1}{2} \lambda_{1}\left(V_{\mu}^{*} V^{\mu}\right)^{2}+\frac{1}{2} \lambda_{2}\left(V_{\mu}^{*} V_{\nu}\right)^{2}+i \kappa V_{\mu}^{*} V_{\nu} F^{\mu \nu}
$$

For the purposes of studying the mass spectrum on the condensate, these terms are not of great importance and we will ignore them within the present section. They are however fully taken into account in the results of section 3 .

The equations of motion, following from (A-I)

$$
\frac{1}{g^{2}} \partial^{\mu} F_{\mu \nu}+i\left(V^{* \mu} G_{\mu \nu}-V^{\mu} G_{\mu \nu}^{*}\right)=J_{\nu}, \quad D^{\mu} G_{\mu \nu}+m_{H}^{2} V_{\nu}=0
$$

possess a solution

$$
A_{\mu}-\partial_{\mu} \alpha=\left(m_{H}, \mathbf{0}\right), \quad V_{\mu}=\left(0, e^{i \alpha} \mathbf{v}\right)
$$

where $\mathbf{v}$ is an arbitrary real vector with the norm $v=\sqrt{J_{0} / 2 m_{H}}$ and $\alpha$ is the overall phase of the vector $V_{\mu}$. The norm of $\mathbf{v}$ is fixed by the first Maxwell equation that enforces the neutrality of the system in the bulk of the condensate. Our choice of vacuum corresponds to $\alpha=0$ :

$$
\left\langle A_{\mu}\right\rangle=\left(m_{H}, \mathbf{0}\right), \quad\left\langle V_{\mu}\right\rangle=(0,0,0, v) \equiv v_{\mu} .
$$

This breaks the local $U(1)$ symmetry, giving mass to the photon. It also breaks the spatial $O(3)$ group, down to $O(2)$. We should therefore expect two NambuGoldstone modes in the spectrum, corresponding to the latter symmetry breaking. 
Hamiltonian of the system (excluding the contribution of the Fermi energy of electrons), on the above background

$$
\mathcal{H}=-\mathcal{L}=m_{H} J_{0},
$$

reduces to the rest energy of the vector bosons.

Let us consider small perturbations of the background (A-V) in the equations of motion (A-III). By setting $V_{\mu}=v_{\mu}+\sigma_{\mu}$ and $A_{\mu}=m_{H} \delta_{\mu}^{0}+a_{\mu}$, one obtains

$$
\begin{gathered}
{\left[\left(\frac{1}{g^{2}} \square+2 v^{2}\right) \eta^{\nu \mu}-\frac{1}{g^{2}} \partial^{\nu} \partial^{\mu}+2 v^{\nu} v^{\mu}\right] a_{\mu}+\left[i v^{\alpha} \partial_{\alpha} \eta^{\nu \mu}-i \partial^{\nu} v^{\mu}\right]\left(\sigma_{\mu}-\sigma_{\mu}^{*}\right)} \\
+\left[m_{H} v^{\nu} \delta_{0}^{\mu}-2 m_{H} \delta_{0}^{\nu} v^{\mu}\right]\left(\sigma_{\mu}+\sigma_{\mu}^{*}\right)=0,(\mathrm{~A}-\mathrm{VII}) \\
{\left[i v^{\alpha} \partial_{\alpha} \eta^{\nu \mu}-i v^{\nu} \partial^{\mu}-2 m v^{\nu} \delta_{0}^{\mu}+\right.} \\
\left.m_{H} \delta_{0}^{\nu} v^{\mu}\right] a_{\mu}+\left[\left(\square-2 i m_{H} \partial_{0}\right) \eta^{\nu \mu}-\partial^{\nu} \partial^{\mu}\right. \\
\left.+i m_{H}\left(\delta_{0}^{\nu} \partial^{\mu}+\partial^{\nu} \delta_{0}^{\mu}\right)+m_{H}^{2} \delta_{0}^{\nu} \delta_{0}^{\mu}\right] \sigma_{\mu}=0, \text { (A-VIII) }
\end{gathered}
$$

where $\eta_{\mu \nu} \equiv \operatorname{diag}(1,-1,-1,-1)$ and all the derivatives act to the right. In order to obtain the mass gaps of perturbations on the condensate, we take the infinite wavelength limit $\left(\partial_{i} \rightarrow 0\right.$, where $\left.i=1,2,3\right)$. It is straightforward to show that the spectrum contains two massless modes, three masive photon degrees of freedom (mass $\sqrt{2} g v$ ), and three modes of mass $2 m_{H}$ which correspond to relatvistic effect of creation of boson-antiboson pair in the condesnate.

\section{Appendix B. Non-Relativistic Limit}

Restoring the factors of $c$, but still keeping $\hbar=1$, gives the following expression for the action

$$
S=\int d t d^{3} x \mathcal{L}=\int d t d^{3} x\left(-\frac{1}{4} F_{\mu \nu} F^{\mu \nu}-\frac{1}{2} G_{\mu \nu}^{*} G^{\mu \nu}+m_{H}^{2} c^{2} V_{\mu}^{*} V^{\mu}-\frac{g}{c} J_{\mu} A^{\mu}\right),(
$$

where the conventional normalization of the photon field is assumed, so that $D_{\mu}=$ $\partial_{\mu}-i g A_{\mu} / c$. The physical quantities are given by the following expressions

$$
\mathbf{E}=-\frac{1}{c} \partial_{t} \mathbf{A}-\nabla A_{0}, \quad \mathbf{B}=\nabla \times \mathbf{A}, \quad \rho=\frac{J^{0}}{c}, \quad j^{i}=J^{i},
$$

where $\mathbf{E}$ and $\mathbf{B}$ are the electric and magnetic fields respectively, $\rho$ denotes the number density of electrons and $\mathbf{j}$ is the corresponding current. The non-relativistic (magnetic) limit is taken by sending $c$ to infinity, in a way that leaves physical quantities finite. This implies the finiteness of $A_{0}, \mathbf{A} / c$ and $J^{0} / c$, and we neglect all quantities, suppressed by an extra factor of $1 / c$. It should be noted, that the 
magnetic field happens to be enhanced by a factor of $c$. We switch to non-relativistic description by the following rescaling of the phase

$$
V^{\mu}=e^{-i m_{H} c x^{0}} W^{\mu}
$$

where $W^{\mu}$ describes the non-relativistic vector field. The Lagrangian therefore becomes

$$
\mathcal{L}=-\frac{1}{4} F_{\mu \nu} F^{\mu \nu}-\frac{1}{2} \Sigma_{\mu \nu}^{*} \Sigma^{\mu \nu}+i m_{H} c W^{* i} \Sigma_{i 0}-i m_{H} c W^{i} \Sigma_{i 0}^{*}+m_{H}^{2} c^{2} W_{0}^{*} W^{0}-\frac{g}{c} J_{\mu} A^{\mu},
$$

where $\Sigma_{\mu \nu}=D_{\mu} W_{\nu}-D_{\nu} W_{\mu}$. As a next step, we take the $c \rightarrow \infty$ limit keeping $c W_{0}$ and $W_{j}$ finite. This is reduces to rescaling $W_{0}$ by a factor of $c$, and after setting $c=1$ again, gives

$$
\begin{array}{r}
\mathcal{L}^{N R}=-\frac{1}{4} F_{\mu \nu} F^{\mu \nu}-\frac{1}{2} \Sigma_{i j}^{*} \Sigma^{i j}+i m_{H} W^{* i} \Sigma_{i 0}-i m_{H} W^{i} \Sigma_{i 0}^{*}+m_{H}^{2} W_{0}^{*} W^{0}- \\
-g J_{\mu} A^{\mu} .
\end{array}
$$

Using the same reasoning, we could also include the rest of dimension-four operators (A-II) in the non-relativistic Lagrangian

$$
\begin{aligned}
\left(\mathcal{L}+\mathcal{L}^{\prime}\right)^{N R}=- & \frac{1}{4} F_{\mu \nu} F^{\mu \nu}-\frac{1}{2} \Sigma_{i j}^{*} \Sigma^{i j}+i m_{H} W^{* i} \Sigma_{i 0}-i m_{H} W^{i} \Sigma_{i 0}^{*}+m_{H}^{2} W_{0}^{*} W^{0}- \\
& \quad-g J_{\mu} A^{\mu}-\frac{1}{2} \lambda_{1}\left(W^{* i} W_{i}\right)^{2}+\frac{1}{2} \lambda_{2}\left(W^{* i} W_{j}\right)^{2}+i g \kappa W^{* i} W^{j} F_{i j} .
\end{aligned}
$$

One can integrate out the nondynamical $W_{0}$ field from the last expression to obtain $(3)^{9}$, which was our starting point for constructing the effective theory of section 2 . The latter form of the Lagrangian makes the connection with Schrödinger's equation explicit.

\section{Appendix C. Spectrum of Perturbations}

In this appendix we briefly summarize the derivation of the full linear spectrum of perturbations on the deuteron condensate. The equations of motion for the perturbations $\left\{\sigma_{0}, a_{0}, \sigma_{\alpha}, \sigma_{3}, a_{\alpha}, a_{3}\right\}$ of the gauge-invariant variables $\left\{W_{0}, A_{0}\right.$, $\left.W_{\alpha},\left|W_{3}\right|, A_{\alpha}, A_{3}\right\}$, which follow from the Lagrangian (6), read as follows:

$$
\begin{array}{r}
-i m_{H} \partial_{\alpha} \sigma_{\alpha}-i m_{H} \partial_{3} \sigma_{3}-m_{H} v a_{3}+m_{H}^{2} \sigma_{0}=0, \\
\frac{1}{g^{2}}\left(-\Delta+m_{0}^{2}\right) a_{0}+\frac{1}{g^{2}} \partial_{0}\left(\partial_{\alpha} a_{\alpha}+\partial_{3} a_{3}\right)+4 m_{H} v \sigma_{3}=0, \\
i m_{H} \partial_{\alpha} \sigma_{0}+\left[\left(-\Delta-2 i m_{H} \partial_{0}\right) \delta_{\alpha \beta}+\partial_{\alpha} \partial_{\beta}\right] \sigma_{\beta}+\partial_{\alpha} \partial_{3} \sigma_{3}-i(1-\kappa) v \partial_{3} a_{\alpha}-i \kappa v \partial_{\alpha} a_{3}+ \\
+\lambda_{s} v^{2}\left(\sigma_{\alpha}-\sigma_{\alpha}^{*}\right)=0,
\end{array}
$$

\footnotetext{
${ }^{9}$ Parameters $\lambda_{1}$ and $\lambda_{2}$ are related to the counterparts of (3) by the following redefinitions $\lambda_{s}=\lambda_{2}, \quad \lambda_{q}=\lambda_{1}-\lambda_{2}$.
} 


$$
\begin{array}{r}
\frac{i}{2} m_{H} \partial_{3}\left(\sigma_{0}-\sigma_{0}^{*}\right)+\left(-\Delta+\partial_{3}^{2}+2 \lambda_{q} v^{2}\right) \sigma_{3}+\frac{1}{2} \partial_{3} \partial_{\alpha}\left(\sigma_{\alpha}+\sigma_{\alpha}^{*}\right)-2 m_{H} v a_{0}=0, \\
-i(1-\kappa) v \partial_{3}\left(\sigma_{\alpha}-\sigma_{\alpha}^{*}\right)-\frac{1}{g^{2}} \partial_{\alpha} \partial_{0} a_{0}+\left(\frac{1}{g^{2}} \square+2 v^{2}\right) a_{\alpha}+\frac{1}{g^{2}} \partial_{\alpha} \partial_{\beta} a_{\beta}+\frac{1}{g^{2}} \partial_{\alpha} \partial_{3} a_{3}=0, \\
m_{H} v\left(\sigma_{0}+\sigma_{0}^{*}\right)-i \kappa v \partial_{\alpha}\left(\sigma_{\alpha}-\sigma_{\alpha}^{*}\right)-\frac{1}{g^{2}} \partial_{3} \partial_{0} a_{0}+\frac{1}{g^{2}}\left(\square+\partial_{3}^{2}\right) a_{3}+\frac{1}{g^{2}} \partial_{3} \partial_{\alpha} a_{\alpha}=0 .
\end{array}
$$

Here $\alpha=1,2$ and $\sigma_{3}$ is real. To obtain the spectrum of perturbations, we find the eigenvalues of the matrix, corresponding to the linear system (C-I)-(C-VI). After a tedious, but straightforward calculation, we find the following expressions for the spectrum

$$
\begin{array}{r}
\omega_{1}^{2}=2 v^{2} g^{2}\left(1+\frac{\mathbf{k}^{4}+2 \mathbf{k}^{2} v^{2} \lambda_{q}}{8 g^{2} m_{H}^{2} v^{2}+\left(\mathbf{k}^{2}+2 v^{2} \lambda_{q}\right) m_{0}^{2}}\right), \\
\omega_{2,4}^{2}=\frac{1}{8 m_{H}^{2}}\left(\mathbf{k}^{4}+8 g^{2} v^{2} m_{H}^{2}+2 \mathbf{k}^{2}\left(2 m_{H}^{2}+v^{2} \lambda_{s}\right) \pm\right. \\
\left.\left(\left(4 m_{H}^{2}\left(2 g^{2} v^{2}+\mathbf{k}^{2}\right)-2 \mathbf{k}^{2} v^{2} \lambda_{s}-\mathbf{k}^{4}\right)^{2}+32 g^{2} m_{H}^{2} v^{2} \mathbf{k}^{4}(1-\kappa)^{2}\right)^{1 / 2}\right), \\
\omega_{3,5}^{2}=\frac{1}{8 m_{H}^{2}}\left(\mathbf{k}^{4}+8 g^{2} v^{2} m_{H}^{2}+2 \mathbf{k}^{2}\left(2 m_{H}^{2}+v^{2} \lambda_{s}\right) \pm\right. \\
\left.\left(\left(4 m_{H}^{2}\left(2 g^{2} v^{2}+\mathbf{k}^{2}\right)-2 \mathbf{k}^{2} v^{2} \lambda_{s}-\mathbf{k}^{4}\right)^{2}+32 g^{2} m_{H}^{2} \mathbf{k}^{2}(\mathbf{k} \cdot \mathbf{v})^{2}(1-\kappa)^{2}\right)^{1 / 2}\right) .
\end{array}
$$

Here, the dispersion relation for the longitudinal polarization of the massive photon is given by $\omega_{1}^{2}$; moreover, $\omega_{2,3}^{2}$ correspond to the transverse polarizations of the photon, and $\omega_{4,5}$ are frequencies for two NG modes. The expressions for $\omega_{3,5}^{2}$ depend on the direction of propagation. Setting $\kappa \in[0,2]$ and $\lambda_{s} \geq 0$, guarantees the absence of instabilities, as can easily be verified.

It should also be noted, that the propagation of one of the NG bosons in the direction perpendicular to $\mathbf{v}$ is independent of the coupling constant $g$ (putting aside the fact that $\lambda_{s}$ itself would be determined by $g$ )

$$
\omega_{5}^{2}=\frac{k^{4}}{4 m_{H}^{2}}+\lambda_{s} \frac{\mathbf{k}^{2} v^{2}}{2 m_{H}^{2}}, \quad \text { for } \quad \mathbf{k} \perp \mathbf{v} .
$$

This curious fact can be understood by a closer inspection of the system (C-VI). Integrating out the auxiliary variable $\sigma_{0}$, as well as the nondynamical heavy modes $\phi_{\alpha} \equiv\left(\sigma_{\alpha}+\sigma_{\alpha}^{*}\right) / 2$, one arrives at a simple equation for the light modes

$$
\left(-\Delta-\frac{4 m_{H}^{2} \partial_{0}^{2}}{\Delta}+2 \lambda_{s} v^{2}\right) \pi_{\alpha}=(1-\kappa) v\left(\partial_{3} a_{\alpha}-\partial_{\alpha} a_{3}\right)
$$

where $\pi_{\alpha} \equiv\left(\sigma_{\alpha}-\sigma_{\alpha}^{*}\right) / 2 i$. It can be easily seen for instance, that for $\mathbf{k}_{2}=\mathbf{k}_{3}=0$ (or $\partial_{2}=\partial_{3}=0$ in position space) $\pi_{2}$ represents the NG field, with the dispersion relation precisely given by (C-VIII), while $\pi_{1}$ mixes with other bosonic fields of the theory, leading to the abovementioned asymmetry between the NG bosons. 


\section{References}

[1] G. Gabadadze and R. A. Rosen, JCAP 0902, 016 (2009) [arXiv:0811.4423 [hepth]]; JCAP 0810, 030 (2008) [arXiv:0806.3692 [astro-ph]]; Phys. Lett. B 658, 266 (2008) [arXiv:0706.2304 [hep-th]].

[2] G. Gabadadze and D. Pirtskhalava, JCAP 0905, 017 (2009) [arXiv:0904.4267 [hep-th]].

[3] G. Gabadadze and R. A. Rosen, arXiv:0912.5270 [hep-ph].

[4] D. G. Hicks et al., Phys. Rev. B 79, 014112 (2009); W. J. Nellis, S. T. Weir, and A. C. Mitchell, Phys. Rev. B 59, 3434 (1999); L. B. Da Silva et al., Phys. Rev. Lett. 78, 483 (1997)

[5] H. B. Nielsen and S. Chadha, Nucl. Phys. B 105, 445 (1976).

[6] D.Q. Lamb and H.M. Van Horn, Ap.J. 200:306 (1975); S. Ichimaru, Rev. Mod. Phys. 54:1017 (1982); G.S. Stringfellow, H.E. DeWitt, W.I. Slattery, Phys. Lett. A 41, 1105 (1990).

[7] A.J. Leggett, "Quantum Liquids," Oxford University Press (2006).

[8] G. Gabadadze, Int. J. Mod. Phys. A 25, 627 (2010) [arXiv:0906.2406 [hep-th]].

[9] A. D. Dolgov, A. Lepidi and G. Piccinelli, JCAP 0902, 027 (2009) [arXiv:0811.4406 [hep-th]]; Phys. Rev. D 80, 125009 (2009) [arXiv:0905.4422 [hep-ph]].

[10] W. Kohn and J. M. Luttinger, Phys. Rev. Lett. 15, 524 (1965).

[11] A. L. Fetter, J. D. Walecka, "Quantum Theory of Many-Particle Systems", McGraw-Hill (1971).

[12] S.L. Shapiro and S. A. Teukolsky, "Black Holes, White Dwarfs, and Neutron Stars", John Wiley and Sons, (1983).

[13] G. Velo and D. Zwanziger, Phys. Rev. 188, 2218 (1969).

[14] M. Porrati and R. Rahman, Nucl. Phys. B 801, 174 (2008) [arXiv:0801.2581 [hep-th]]. 